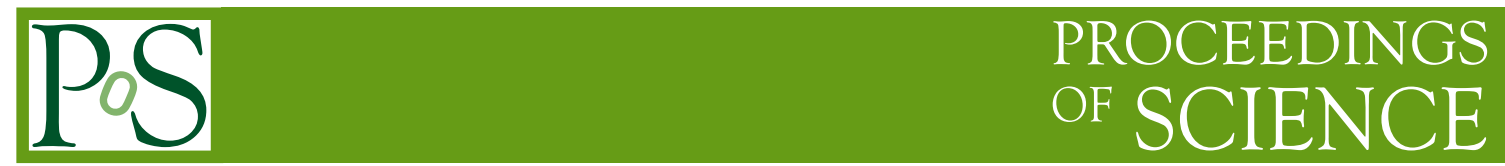

\title{
Tau lifetime and CP violation in tau decay at Belle
}

\section{Mikhail Shapkin*t}

IHEP, Protvino, Russia

E-mail: Mikhail.Shapkindihep.ru

The method of the $\tau$-lepton lifetime measurement where the present accuracy may be improved by a factor about two is reported. The value of an upper limit on the relative lifetime difference between positive and negative $\tau$-leptons is obtained. The results of the $\mathrm{CP}$ violation search in the $\tau^{ \pm} \rightarrow K_{S} \pi^{ \pm} v_{\tau}$ decays are reported. The upper limit on the CP violating coupling constant is obtained. The obtained results are based on $700 \mathrm{fb}^{-1}$ of data collected at the $\Upsilon(4 S)$ resonance.

35th International Conference of High Energy Physics - ICHEP2010,

July 22-28, 2010

Paris France

\footnotetext{
* Speaker.

†n behalf of the Belle Collaboration
} 
High precision measurements of the mass, lifetime and leptonic branching fractions of the $\tau$-lepton can be used to test the lepton universality [1], which is assumed in the Standard Model. The present PDG value of the $\tau$ lifetime [2] is dominated by the results obtained by LEP experiments [3]. BABAR experiment has also reported its preliminary result based on the data sample 80 $\mathrm{fb}^{-1}$ [4] which is consistent with the PDG value and has the same level of precision as in the PDG. A high statistics data sample collected at Belle allows to select $\tau^{+} \tau^{-}$events where both $\tau$-leptons decay to three charged pions and neutrino. The Belle $\tau$-lepton lifetime precision measurement has systematic uncertainties quite different from the LEP experiments, that is why the combined result can be used for the test of the hypothesis of the lepton universality. The present analysis allows to obtain the lifetimes of positive and negative $\tau$-leptons separately and test the CPT theorem.

To date CP violation (CPV) has been observed only in meson systems. In the leptonic sector $\mathrm{CPV}$ is not allowed within the Standard Model (SM). Hence, its discovery would be clear evidence for a New Physics (NP). In hadronic $\tau$ decays CPV can occure due to the interference between the SM W boson mediated and a NP boson mediated decays to the same final state. This paper describes a search for CPV in $\tau^{ \pm} \rightarrow K_{S} \pi^{ \pm} v_{\tau}$ decays. It should be noted that because of CPV in $K^{0}$ decays a small SM CP asymmetry of $\mathrm{O}\left(10^{-3}\right)$ in this decay should be for the full rate [5]. Here, instead, the focus will be CPV in context of a model with a charged scalar boson exchange [6]. This type of CPV can be observed as a difference in the decay angular distributions and is accessible without requiring information about the $\tau$ polarisation.

\section{1. $\tau$-lepton lifetime analysis}

If we assume the neutrino mass to be zero for the hadronic decay $\tau \rightarrow X v_{\tau}$ (X is the hadronic system) the angle $\theta$ between the momentum of the system $X$ and the momentum of the $\tau$-lepton is determined as $\cos (\theta)=\frac{2 E_{\tau} E_{X}-m_{\tau}^{2}-m_{X}^{2}}{2 P_{X} \sqrt{E_{\tau}^{2}-m_{\tau}^{2}}}$. The requirement that $\tau$-leptons go back to back in the $\mathrm{CM}$ frame may be written as a system of two linear and one quadratic equations. Two solutions of this system of equations are possible for $\tau$-lepton flight directions. In order to solve this two-fold ambiguity, we go to the Laboratory frame. In this frame $\tau$-leptons should go along the straight lines which intersect in the same space point. Due to the finite detector resolution the straight lines do not intersect in the 3-dimensional space. The separation between them is characterized by the distance $d l$. For the true solution, which defines the $\tau$-lepton direction in the CM frame, we expect the smaller value of $d l$ than for the wrong one. This assumption is supported by the Monte Carlo (MC) simulation of $\tau^{+} \tau^{-}$events. Thus, for each $\tau^{+} \tau^{-}$event, where both $\tau$-leptons decay into a hadronic system and neutrino and where the $\tau^{+}, \tau^{-}$decay vertices are reconstructed in the Laboratory frame we can unambiguously determine the lifetime parameters $c \tau$ for both $\tau$-leptons. Below we will analyze $\tau^{+} \tau^{-}$events, where both $\tau$-leptons decay to 3 charged pions and neutrino. The $c \tau$ distribution for the MC $\tau^{+} \tau^{-}$events were fitted by the convolution of the exponential function with the resolution function $\mathrm{R}(\mathrm{x})$. For the data description we introduce a free parameter $\delta$ which allows broadening the resolution: $R(x) \rightarrow R(x(1+\delta))$. In order to treat the data and $\mathrm{MC}$ in the same way we fitted both the data and $\tau^{+} \tau^{-} \mathrm{MC} c \tau$ distributions by the convolution of the exponential function and modified resolution function $R(x(1+\delta))$ with three free parameters corresponding to the normalization, $\tau$ lepton lifetime and broadening of the resolution function. In the data the contamination of the background is about a few percent. The main source of the 


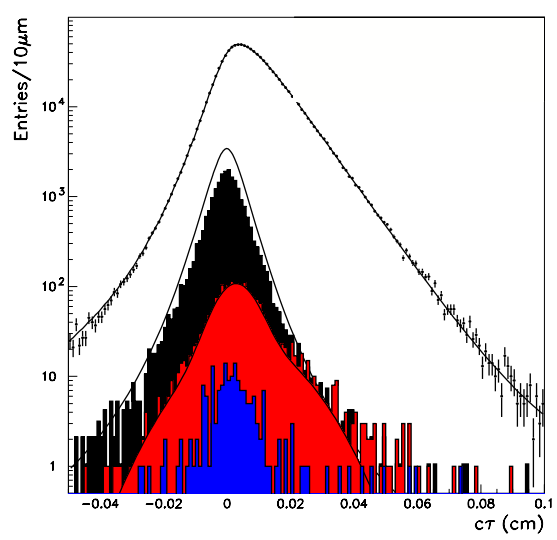

Figure 1: The reconstructed $c \tau$ values for the data (filled circles with errors). The line which goes through the experimental points is the result of the fit. The gray, red and blue filled histograms are the MC predictions for the sum of $u d s$ and $\gamma \gamma$, charm and beauty events, respectively. The line which goes higher than the filled gray histogram is the $u d s$ contribution with the fixed $A_{u d s}$ parameter which was used in the fitting the data.

background is $u d s$ events. For these events, all six pions come mainly from one primary vertex and they are similar to the $\tau^{+} \tau^{-}$events with the zero lifetime. That is why we expect that the reconstructed $c \tau$ distribution for these events may be described by the resolution function $R(x)$. The same behavior is expected for $\gamma \gamma$ events. This assumption is supported by MC. Other sources of the background contribute to the selected data sample at the per mille level. They were fixed from MC prediction. The experimental $c \tau$ distribution together with the result of the fit and estimated sources of background contributions are shown in Figure 1. The statistical accuracy of $c \tau$ parameter obtained from the fit is $0.11 \mu \mathrm{m}$ or $0.37 \mathrm{fs}$. The analysis of systematic uncertainties is not finished yet. We analysed the uncertainties due to limited MC statistics, choice of the fit range of the experimetal distributions, accuracy of the initial and final state radiation by $\mathrm{MC}$, knowledge of the beam energy, alignment of the vertex detector, knowledge of background contribution, error of the $\tau$ lepton mass. The overall systematic uncertainty turns out to be smaller than the statistical uncertainty $(0.33 \mathrm{fs})$. The precent uncertainty of mean PDG value is $1.0 \mathrm{fs}$ [⿰]. lifetimes for $\tau^{+}$and $\tau^{-}$separately and found that they are equal within the statistical accuracy. Because the most systematic ancertainties cancell in the difference of lifetimes the $90 \%$ upper limit is $\left|\tau_{\tau^{+}}-\tau_{\tau^{-}}\right| / \tau_{\text {average }}<6.0 \cdot 10^{-3}$.

\section{CP violation in $\tau^{ \pm} \rightarrow K_{S} \pi^{ \pm} v_{\tau}$ decays}

For the analysis the 1-3 topology of the $\tau^{+} \tau^{-}$decays is selected (in the CM frame the event is divided into two hemispheres using the plane perpendicular to the direction of the thrust axis, one hemisphere should contain one charged track from electron, muon or pion, the other hemisphere should contain charged pion and a $K_{S} \rightarrow \pi^{+} \pi^{-}$candidate with an invariant mass in the range 0.485$0.511 \mathrm{GeV} / c^{2}$ and reconstructed $K_{S}$ decay length greater than $\left.2 \mathrm{~cm}\right)$. In total, $(162.2 \pm 0.4) \times$ $10^{3} \tau^{+} \rightarrow K_{S} \pi^{+} \bar{v}_{\tau}$ and $(162.0 \pm 0.4) \times 10^{3} \tau^{-} \rightarrow K_{S} \pi^{-} v_{\tau}$ candidates are selected. The main 


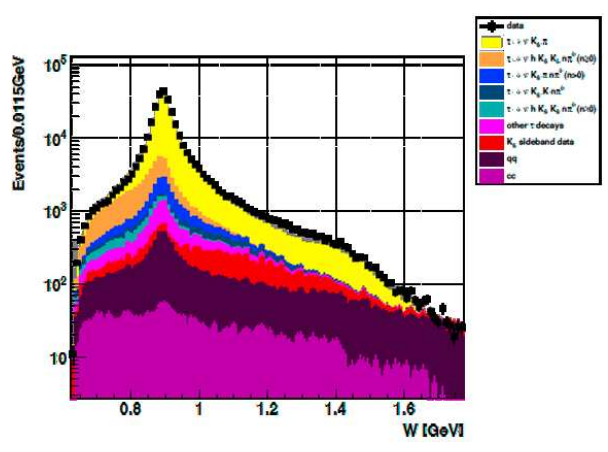

Figure 2: Mass spectrum of the $K_{S} \pi^{ \pm}$system. Data is indicated by squares, background contributions are shown by colored histograms.

background contribution is due to other $\tau$ decays, namely $(9.5 \pm 3.2) \%$ from $\tau^{ \pm} \rightarrow v_{\tau} K_{S} K_{L} \pi^{ \pm}$, $(3.7 \pm 1.2) \%$ from $\tau^{ \pm} \rightarrow v_{\tau} K_{S} \pi^{ \pm} \pi^{0},(1.7 \pm 0.2) \%$ from $\tau^{ \pm} \rightarrow v_{\tau} K_{S} K^{ \pm}$and $(1.79 \pm 0.03) \%$ from $\tau^{ \pm} \rightarrow v_{\tau} \pi^{ \pm} \pi^{+} \pi^{-}$. Contributions from $q \bar{q}$ are $(3.4 \pm 1.0) \%$. The mass spectrum of the $K_{S} \pi^{ \pm}$ system, W, is shown in Figure 2. To extract CP violating parameter $\eta_{S}$ (see the defintion in paper [6]), the asymmetry is measured in bins of $\mathrm{W}$ as difference of integrals over the angles of the decay products for $\tau^{-}$and $\tau^{+}$normalized to the sum of the $\tau^{+}$and $\tau^{-}$in the given interval of W. In order to take into account for possible systematic uncertainties due to unknown detector effects, the values of asymmetry were measured for the control sample $\tau^{ \pm} \rightarrow v_{\tau} \pi^{ \pm} \pi^{+} \pi^{-}$. The $\mathrm{CP}$ asymmetry for the control sample is zero within statistical errors, so these errors are used as systematic uncertainties estimate. No significant CP asymmetry has been observed. The upper limits on the imaginary part of $\eta_{S}$ at $90 \%$ confidence level are $\left|\operatorname{Im}\left(\eta_{S}\right)\right|<0.026$ or better, depending on the parametrization used to describe the hadronic form factors. They improve the previous limits [7] by one order of magnitude.

\section{References}

[1] Y.S. Tsai, Phys.Rev. D 4, 2821 (1971);

H.B. Thacker and J.J. Sakurai, Phys. Lett. B 36, 103 (1971).

[2] C. Amsler et al. (Particle Data Group), Phys. Lett. B 667, 1 (2008)

[3] P. Abreu et al. (DELPHI Collaboration), Phys. Lett. B 365, 448 (1996);

G. Alexander et al. (OPAL Collaboration), Phys. Lett. B 374, 341 (1996);

R. Barate et al. (ALEPH Collaboration), Phys. Lett. B 414, 362 (1997);

M. Acciarri et al. (L3 Collaboration), Phys. Lett. B 479, 67 (2000).

[4] A. Lusiani, Nucl. Phys. B (Proc. Suppl.) 144, 105 (2005).

[5] I.I. Bigi and A.I.Sanda, Phys. Lett. B 625, 47 (2005); G.Calderon, D.Delepine and G.L.Castro, Phys. Rev. D 75, 076001 (2007).

[6] J.H.Kuhn and E.Mirkes, Phys. Lett. B 398, 407 (1997);

[7] P. Bonvincini et al. (CLEO Collaboration), Phys. Rev. Lett. B 88, 111803 (2002); 